\title{
Stomach simulator for analysis and validation of surgical endoluminal robots
}

\author{
Sara Condino ${ }^{a, *}$, Kanako Harada ${ }^{b}$, Nicola Ng Pak ${ }^{b}$, Marco Piccigallo ${ }^{b}$, Arianna Menciassi ${ }^{b}$ \\ and Paolo Dario ${ }^{\mathrm{b}}$ \\ ${ }^{a}$ EndoCAS Center, Dipartimento di Oncologia dei Trapianti e delle Nuove Tecnologie in Medicina, \\ University of Pisa, Pisa, Italy \\ ${ }^{\mathrm{b}}$ The Biorobotics Institute, Scuola Superiore Sant'Anna, Pisa, Italy
}

\begin{abstract}
A testing environment that imitates gastric geometry and contractile activity is necessary to analyse and validate endoluminal surgical robotic devices developed for gastric pathologies. To achieve this goal, a silicone stomach model and a mechanical setup to simulate gastric contractile motion were designed and fabricated. The developed stomach simulator was validated and its usefulness was demonstrated by means of internal pressure measurements and self-assembly tests of mock-ups of capsule devices. The results demonstrated that the stomach simulator is helpful for quantitative evaluation of endoluminal robotic devices before in-vitro/in-vivo experiments.
\end{abstract}

Keywords: Stomach simulator, testing environment, gastric peristaltic activity simulation, robotic endoluminal surgery

\section{Introduction}

The trend of minimally invasive surgery has led to the development of new endoluminal devices. Miniaturized robotic devices have been studied including capsule endoscopes with locomotion [10, 17, 18], swimming capsule endoscopes [19] and robots for NOTES (Natural Orifice Transluminal Endoscopic Surgery) [12]. An assembling reconfigurable endoluminal surgical (ARES) system has been also proposed [6], and in the proposed system, robotic capsules are ingested by a patient and assemble in the stomach filled with a liquid to configure a robotic topology, and then the robot changes its configuration to another topology for diagnosis and intervention in the gastrointestinal (GI) tract (Fig. 1).

For the design and preliminary tests of such endoluminal robotic devices, test benches replicating the

\footnotetext{
*Corresponding author: Sara Condino, EndoCAS Center, Università di Pisa, Ospedale di Cisanello, Via Paradisa 2, 56124 Pisa, Italy. Tel.: (+39) 050995 68925; Fax: (+39) 050995 676; E-mail: sara.condino@endocas.org.
}

GI tract geometry and functionality are necessary. For instance, the success ratio of the self-assembly of the robotic capsules in the stomach or the control of the swimming capsules must be influenced by the size/shape of the stomach and its contractile motion.

In most studies in surgical robotics, in-vitro and invivo animal experiments take the main role to evaluate the performance of surgical devices. However, it is difficult to obtain quantitative and reproducible data due to individual differences of the animal organs and difficulty to control their motions. Regarding the stomach for which some of the endoluminal surgical devices are being developed, the available stomach models are limited to plastic models for medical education, silicone models $[7,8]$ that replicate the gastric anatomy but do not provide any simulation of the stomach physiologic activity, or electromechanical models that simulate the gastric activity but do not have a realistic shape [14].

A simulator replicating the GI tract geometry and functionality can give an alternative to the use of animal organs. In addition, it enables repeatable quantitative evaluation of the developed surgical devices under controlled condition. Hence, the aim of the present study 

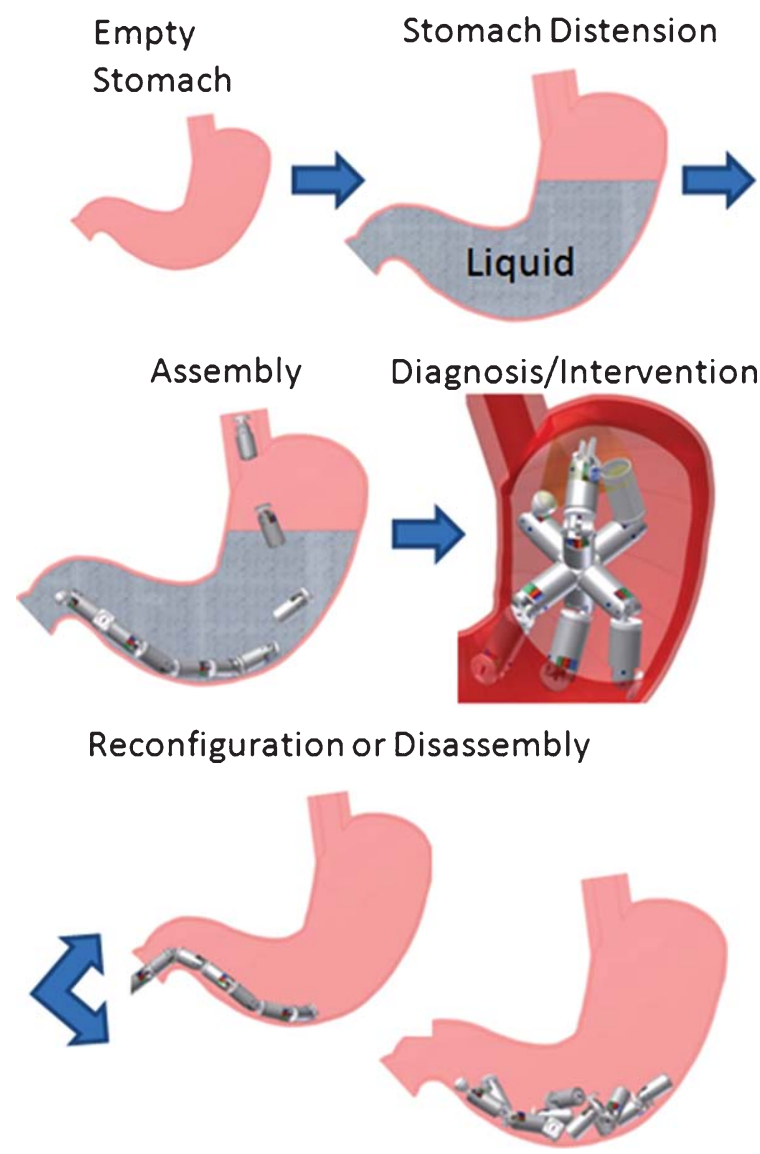

Fig. 1. An assembling reconfigurable endoluminal surgical (ARES) system.

is to design and fabricate a stomach simulator that can replicate the gastric geometry and contractile motion to be used as a test bench for endoluminal surgical robots. Self-assembly tests of simple modular mock-ups were conducted by using the prototyped stomach simulator to evaluate its usefulness.

\section{Stomach features}

Basic understanding of the target organ was fundamental for the definition of the main requirements of the stomach simulator and for the extraction of the specifications needed for the subsequent engineering design. In the following subsections, main characteristics of the stomach are discussed from a mechanical viewpoint and summarized in a quantitative manner so that the data would be useful for other developments as well.

\subsection{Dimensions}

The stomach is a hollow organ whose shape resembles an oblong bag. It lies crosswise in the abdominal cavity beneath the diaphragm, to the right of the spleen and partially under the liver.

Two valves, called sphincters, keep the food inside the stomach contained. These sphincters are the cardiac sphincter (also known as the lower esophageal sphincter) dividing the stomach from the esophagus, and the pyloric sphincter dividing the stomach from the small intestine.

Anatomically, the stomach can be divided into three major regions: fundus, corpus and antrum according to their features.

The stomach changes size and shape according to the posture of the body, the amount of food inside, the stage which the digestive process has reached and the condition of the adjacent intestines. The volume of the stomach of an adult can vary between $0.27-0.39$ litres in an empty state according to the data obtained by MR imaging [3], and the gastric capacity in a healthy person ranges between 0.7 and 1.6 litres [2]. The maximum volume of the stomach can reach up to 4 litres [9].

The structure of gastric wall follows the typical organization of the GI wall, and it consists of four layers: the mucosal (the innermost), the submucosa, muscolaris and the serosal (the outermost). At rest, the inner surface of stomach is characterized by the gastric folds. On the basis of abdominal computer tomography the mean gastric wall thickness is between 2.35 and $5.43 \mathrm{~mm}$ [8]

\subsection{Contractile motion}

The stomach can be divided into two main sections with respect to its motility; the proximal section is devoted to the collection of food, and the distal section mixes and propels the food toward the small intestine.

The contractile motion of the stomach consists of a tonic contraction along the entire length and a peristaltic contraction mainly located in the distal section. The tonic contraction produces a variation of the internal pressure of about $10 \mathrm{mmHg}$, while the peristaltic contraction changes the internal pressure from 3 to $30 \mathrm{mmHg}$ in the distal part with different pressure ranges in each person [1]. Gastric slow waves are the electrical events that control gastric contraction and they result from a spontaneus depolarization of the interstitial cells of Cajal (ICC) in the pacemaker area, 
a region in the gastric greater curvature at the junction of the fundus and body.

They move circumferentially and distally toward the distal antrum. The slow wave controls the timing and propagation of peristaltic contraction which has the frequency of 3 cycles per minute [11] and the traveling speed ranging from 3 up to $6 \mathrm{~mm} / \mathrm{s}$ [4].

\section{Design and fabrication of the stomach simulator}

The aim of developing a stomach simulator is to set up a stomach model simulating the gastric geometry and contractile motion.

A straightforward approach is to design an elastic stomach model and a mechanical setup to contract it by synchronizing the preset parameters of the contractile motion (calculated as described in paragraph 3.3). A silicone rubber material, which allows an easy reproduction of objects with complex shapes and has faithfulness in the reproduction of details, has been chosen for the elastic model fabrication. After the silicone stomach model was fabricated, stainless wire ropes were placed around the silicone stomach model so that each rope can contract a section of it. In the following, the design and fabrication of the silicone stomach model and the mechanical setup of the contraction system are described.

\subsection{Silicone stomach model}

The shape of the silicone stomach model needs to be anatomically correct. Therefore, a plastic anatomical model ordinarily employed for medical education (Stomach model K15, 3B Scientific, Germany) was used as a reference.

This plastic model represents a human empty stomach with an internal volume of 0.44 litres (measured), and it was used to estimate the dimensions of the stomach at rest: firstly, the model was molded in silicone and the silicone mold was cut into several sections to measure the model dimensions. Thereafter, a 3D CAD model of the stomach with a simplified anatomy was created based on the measured dimensions, and it was uniformly enlarged up to the volume of 1.4 litres in agreement with data by Csendes et al. mentioned in the Section 2.1. Using this CAD model, a mold and a core were fabricated using a 3D rapid prototyping machine (Invison XT 3-D Modeler, 3D systems, Inc., USA).
Finally, a silicone stomach model was fabricated by simply filling the mold with liquid silicone rubber. The silicone used in this fabrication is Silastic ${ }^{\circledR}$ (No. 3483, Dow Corning, USA) with low hardness (Shore A = 13) and high elongation at break $(600 \%)$ that allows peeling away the silicone stomach model from the core without damaging it. These fabrication procedures are summarized in Fig. 2.

\subsection{Design of the mechanical setup of the contraction system}

A cables actuation system was designed for imitating the gastric contractile activity. In particular, it was designed to reproduce the gastric musculature action by placing miniature stainless wire ropes on the model outer surface.

Retaining structures for the wire ropes, as the one illustrated in Fig. 3, were fabricated using polyurethane (PC26, Elantas Camattini, Italy). These retaining structures were attached to the surface of the silicone stomach model using cyanoacrylate (LOCTITE ${ }^{\circledR}$ $406^{\mathrm{TM}}$, Henkel, Germany) and polyolefin primer (LOCTITE $^{\circledR} 770^{\mathrm{TM}}$, Henkel, Germany). The wires exposed between the attached retaining structures were covered with polyurethane tubes to avoid damage of the silicone by the wires.

A slider actuated with a brushed DC motor was designed to pull each wire rope placed around the silicone stomach model (Fig. 4). Springs are used to push the slider back when the wire is released. The elasticity of the silicone enables the stomach model to restore its original dilated shape when the wire is released.

\subsection{Control of the contractile motion}

The next step is to establish a control method to reproduce the gastric contractile motion.

A quantification of the degree of gastric lumen occlusion during the contractile motion is necessary for the calculation of the wire rope pulling speed and distance.

In this study, the data reported by Pal et al. [16] was used as a reference. In their paper, gastric flow and mixing was studied using computer simulation, and a realistic geometry model of the stomach was created by using MRI data. The parameters for gastric motility were obtained from the data in addition to the parameters for the timing and location of gastric 


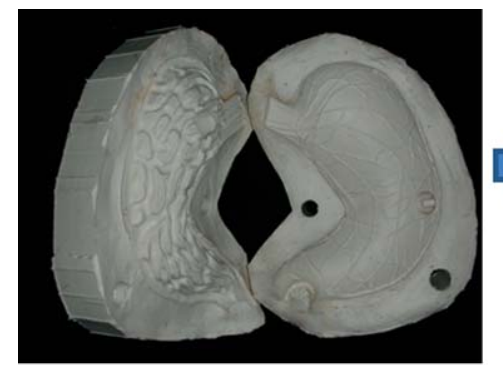

Moulded parts using a plastic anatomical model (Internal volume: $0.44 \mathrm{~L}$ )

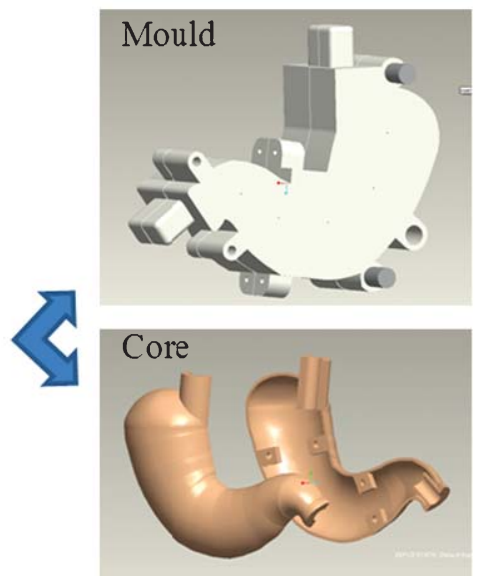

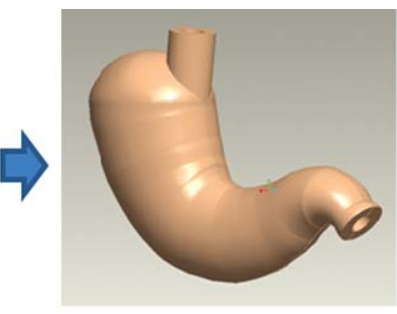

CAD model of an empty stomach (Internal volume: $0.44 \mathrm{~L}$ )

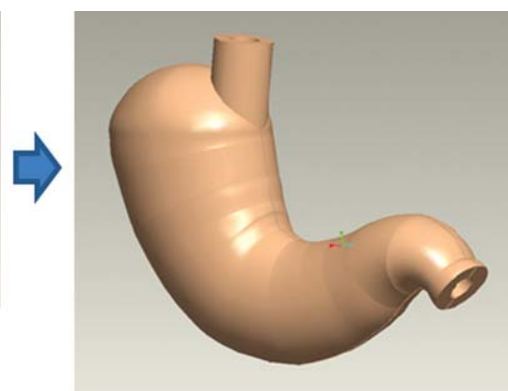

CAD model of a full stomach (Internal volume: $1.4 \mathrm{~L}$ )
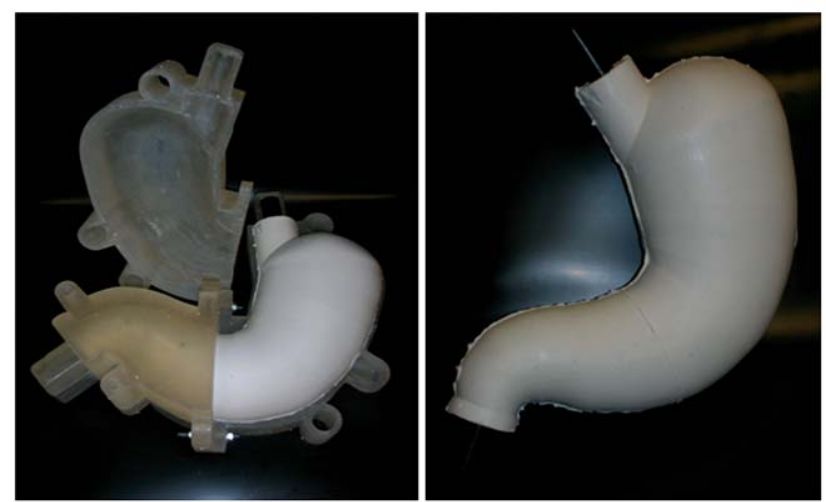

Fabricated silicone stomach models

Fig. 2. Fabrication procedures of a silicone stomach.

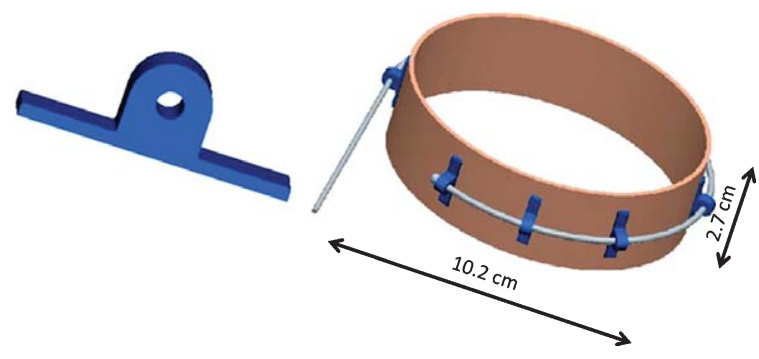

Fig. 3. Retaining structure and example of silicone cylindrical structure to be contracted with the designed cables actuation system.

motility events. They defined the relative occlusion of ACW (antral contraction wave) as:

$$
\alpha=\frac{r}{R}
$$

where $\alpha$ is the relative occlusion of ACW, $r$ is the occlusion radius and $R$ is the radius without contraction.

In their model, the gastric area where peristalsis occurs (gastric body and antrum) can be divided in

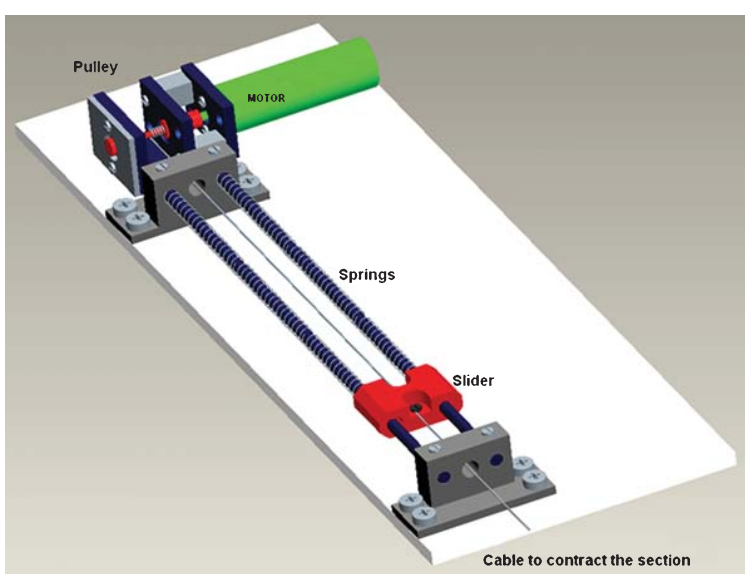

Fig. 4. Slider to actuate a miniature stainless wire rope.

three regions depending on the level of ACW occlusion. In the proximal region ranging from 140 to $100 \mathrm{~mm}$ of distance to the pylorus, the relative occlusion of ACW $(\alpha)$ decreases linearly from 1 to 0.6 . In 
the middle region ranging from 100 to $60 \mathrm{~mm}$ of distance to the pylorus, the relative occlusion of ACW $(\alpha)$ remains constant with a value of 0.6 . In the distal region, the relative occlusion of $\mathrm{ACW}(\alpha)$ decreases linearly reaching 0.1 at the pylorus.

In the present paper, the data was adapted to the model of the enlarged stomach (1.4 litres) for quantifying the degree of its lumen occlusion during the peristalsis simulation. Three regions similar to the ones described by Pal et al. were identified in the 3D CAD model: the region No. 1 from 231 to $165 \mathrm{~mm}$ of distance to the pylorus, the region No. 2 from 165 to $99 \mathrm{~mm}$ of distance to the pylorus and the region No. 3 that terminates at the stomach distal sphincter. All the distances were measured along the 3D CAD model center line. Four cables were then placed on the outer surface of the stomach model, in correspondence of the four sections that delimit the above mentioned regions. As showed in Fig. 5, the last cable was placed at the $2 / 3$ of the distal region in order to have four equally spaced contracting sections. The relative occlusion of ACW $(\alpha)$ was defined for every section according to its position inside the 3D model and the occlusion radius was consequently calculated.

Regarding the force assessment for the control of the contractile motion, the necessary force of the motor in the mechanical setup was estimated by supposing that the motor needs to pull the wire rope against three different forces: the force related to the inner pressure increases during the contraction, the force of the springs, and the resistance of the silicone wall to corrugation.

To estimate the force related to the increase of the internal pressure, the portion of stomach model con- tracted by each cable was modelled as a cylinder as shown in Fig. 5(b). The stomach has an inner pressure change of maximum $30 \mathrm{mmHg}$ during peristaltic motion as reported by Berne-Levy [1]. According to Laplace's law, the tension $(T)$ in a thin-walled cylindrical structure can be defined as:

$$
T=\Delta p \cdot r
$$

where $\Delta p$ is transmural pressure and $r$ is radius of the structure. This law is valid for thin-walled structure, assuming that the wall tension is constant everywhere and that the material property is isotropic, but it is considered a good approximation to calculate tension in tubular anatomies as the stomach [5]. Starting from equation (2) the force needed for each motor to compensate the inner pressure change $\left(F_{p i}\right)$ can be modelled as :

$$
F_{p i}=\Delta p \cdot r_{i} \cdot h
$$

where $i$ is an index that representing the section number that varies from 1 to 4 (from proximal to distal as shown in Fig. 5) and $\Delta p\left[\mathrm{~N} / \mathrm{mm}^{2}\right], r_{i}[\mathrm{~mm}]$ and $h[\mathrm{~mm}]$ are respectively the variation of the inner pressure, the occlusion radius and the height of the modelled cylinder. Next, the force by the springs in the mechanical setup was calculated by considering the maximum contraction of each cylinder, which determines the maximum displacement of the slider in the setup. The maximum displacement $L_{i}[\mathrm{~mm}]$ of each slider can be described as:

$$
L_{i}=2 \pi \cdot\left(R_{i}-r_{i}\right)=2 \pi R_{i} \cdot\left(1-\alpha_{i}\right)
$$

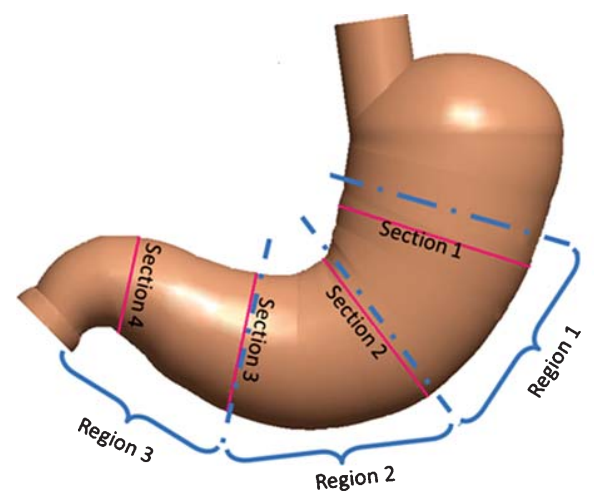

(a)

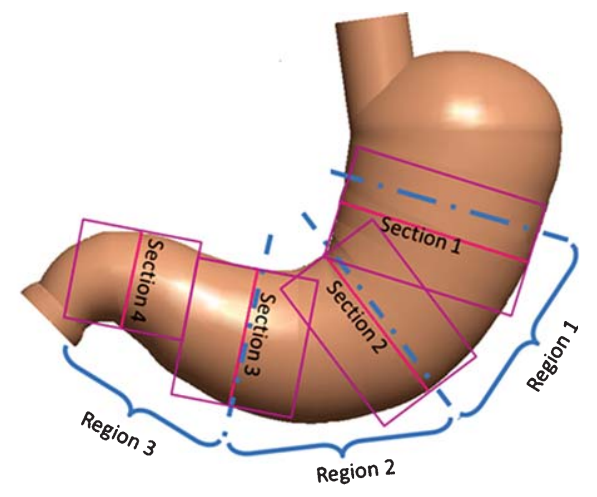

(b)

Fig. 5. (a) Stomach regions and sections in correspondence to the positioned cables. (b) Cylindrical volume modelled for each contraction ring. 


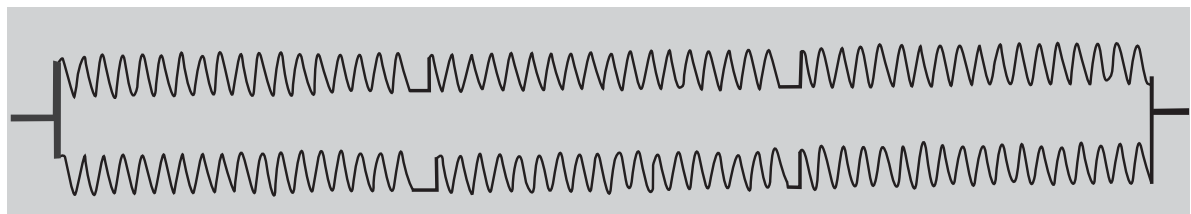

Fig. 6. Model of the springs placed in each slider.

where $R_{i}$ is the radius of the cylinder without contraction (measured in the 3D CAD model of the enlarged stomach). A series of six springs were set in each slider as illustrated in Fig. 6: we should underline that any mechanical setup is acceptable as long as it satisfies the requirements about the displacement and force. In the setup designed using six springs, the maximum force to pull the wire rope against the force by the springs $\left(F_{S_{i}}\right)$ is described as:

$$
F_{s_{i}}=\frac{2}{3} k \cdot L_{i}
$$

where $k[\mathrm{~N} / \mathrm{mm}]$ is $0.11[\mathrm{~N} / \mathrm{mm}]$.

The thickness of the silicone stomach wall was determined to be $2 \mathrm{~mm}$, as an approximation of the mimimum value of the gastric wall thickness according to Henry et al. [8]. Finally the resistance of the silicone has been experimentally determined and the force needed for all the motors to corrugate the stomach model $\left(F_{c}\right)$ was approximated to be $2 \mathrm{~N}$.

On the basis of the above mentioned assumptions, the total pulling force necessary for each motor $\left(F_{i}\right)$ is expressed by adding the three mentioned contributions:

$$
F_{i}=F_{p_{i}}+F_{s_{i}}+F_{c}
$$

Regarding the speed and timing of the motor actuation, the displacement $x_{i}[\mathrm{~mm}]$ of each slider at the time $t[\mathrm{~s}]$ is described as:

$x_{i}=0 \quad t<t_{d_{i}}$

$x_{i}=\frac{L_{i}}{2} \cdot \sin \left(2 \pi \cdot f \cdot\left(t-t_{d_{i}}\right)-\frac{\pi}{2}\right)+\frac{L_{i}}{2} \quad t \geq t_{d i}$

where $f$ is the frequency of the contractile motion and was set as 3 cycles/min. The parameter $t_{d_{i}}[\mathrm{~s}]$ is the delay time between the beginning of contraction of two consecutive sections and it is described as:

$$
t_{d i}=(i-1) \cdot \frac{h}{V}
$$

where $V$ is the wave travelling speed which was set as $2.5 \mathrm{~mm} / \mathrm{s}$. The speed of the slider $v_{i}[\mathrm{~mm} / \mathrm{s}]$ is described as:

$$
v_{i}=\frac{\partial x_{i}}{\partial t}=\pi \cdot L_{i} \cdot f \cdot \cos (2 \cdot \pi \cdot f \cdot t)
$$

And the maximum speed of the slider $v_{i \max _{i}}$ is expressed as:

$$
v_{i \max }=\pi \cdot L_{i} \cdot f
$$

Using these data and considering that the pulley connected to the wire rope has a diameter of $2 \mathrm{~mm}$ and the cable has a diameter of $0.3 \mathrm{~mm}$, the torque and the power needed for the motor was calculated and a geared DC brushed motor (1516-006 SR, Faulhaber, Germany) was selected. A PID algorithm was used to control motors positions via PC serial port, and custommade drivers [13] were used in the experiments. It is worth noting that the time response is not crucial for this setup because the stomach peristaltic motion is as slow as three times per minute. Table 1 shows the parameters defined and derived in this section.

Table 1

Parameters of each stomach model section

\begin{tabular}{lcccc}
\hline Section number $i$ & 1 & 2 & 3 & 4 \\
\hline $\begin{array}{l}\text { Radius of the section } R \\
\quad[\mathrm{~mm}]\end{array}$ & 52.1 & 44.5 & 36.8 & 24.5 \\
$\begin{array}{l}\text { Height of the odelled } \\
\quad \text { cylinder } h \text { [mm] }\end{array}$ & 66.0 & 66.0 & 66.0 & 66.0 \\
$\begin{array}{l}\text { Relative occlusion of } \\
\quad \text { ACW } \alpha\end{array}$ & 0.9 & 0.6 & 0.6 & 0.2 \\
$\begin{array}{l}\text { Maximum displacement } \\
\text { of the slider } L[\mathrm{~mm}]\end{array}$ & 32.7 & 111.8 & 92.5 & 123.1 \\
$\quad F_{p}[\mathrm{~N}]$ & & & & \\
$\quad F_{s}[\mathrm{~N}]$ & 2.06 & 1.17 & 0.97 & 0.21 \\
$\quad F_{c}[\mathrm{~N}]$ & 2.40 & 8.20 & 6.78 & 7.71 \\
$\quad F[\mathrm{~N}]$ & 2.00 & 2.00 & 2.00 & 2.00 \\
Maximum speed of the & 6.46 & 11.38 & 9.75 & 9.03 \\
$\quad$ slider $V_{\text {i max }}[\mathrm{mm} / \mathrm{s}]$ & 5.1 & 17.6 & 14.5 & 19.3 \\
& & & & \\
\hline
\end{tabular}




\section{Experimental methods}

\subsection{Validation of the stomach simulator}

To validate the contractile motion of the stomach simulator, the internal pressure was measured using a pressure sensor (40PC001B1A, Honeywell, USA). The experimental setup of the stomach simulator is shown in Fig. 7, while Fig. 8 illustrates its motion during one cycle of contraction.

First, each section of the stomach model was contracted independently for 3 minutes then the variation of the internal pressure was measured. This experiment has been carried out to confirm that every section is adequately contracted. Next, all sections were contracted based on the programmed timing, and the internal
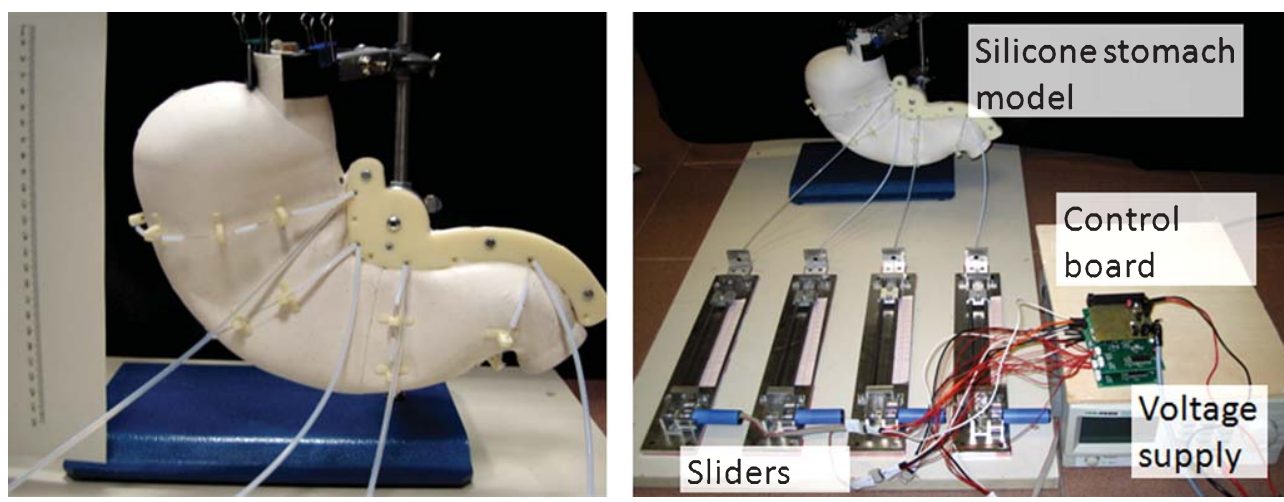

Fig. 7. Experimental setup of the stomach simulator.
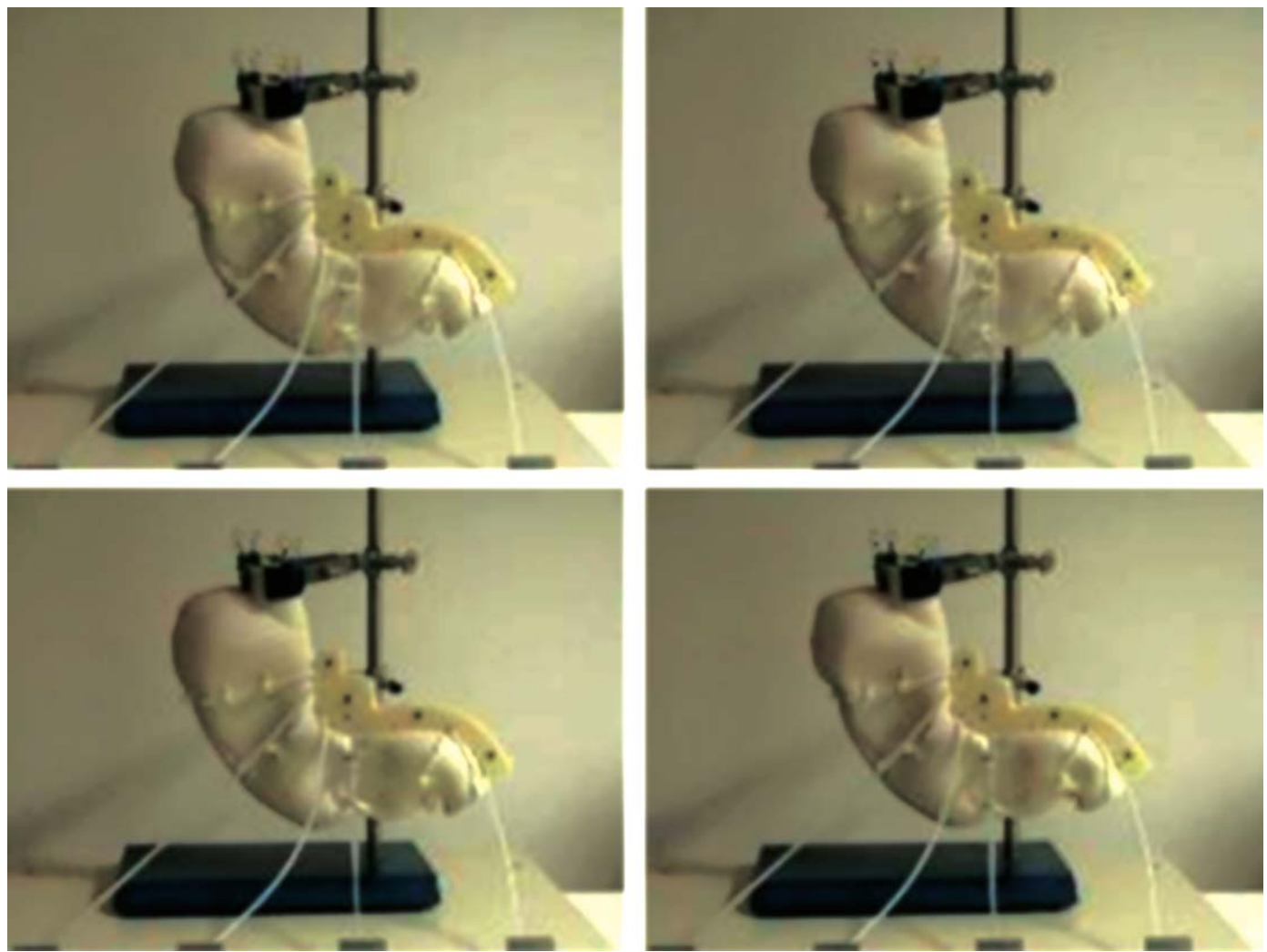

Fig. 8. Motion of the stomach simulator. 
pressure variation of the synchronized contraction was measured for 3 minutes.

\subsection{Assembly tests}

The first demonstration using the developed stomach simulator is to test self-assembly of the modular mockups proposed by the ARES system as described in the introduction.

The idea of the modular self-assembly is to use permanent magnets placed at both ends of the robotic capsule for the self-assembly in liquid. The related work [15] reports preliminary results of magnetic selfassembly by using the passive acrylic stomach model whose shape and dimension are same as the stomach model in the present paper. The objective of the present study is to use the stomach simulator to estimate the influence of the gastric contractile motion to the success ratio of the magnetic self-assembly, especially compared to the success ratio measured by using the acrylic stomach model. As shown in Fig. 9, mockups with two magnets at both ends were designed and fabricated for testing purposes. Because this kind of experiments cannot be performed using existing stomach models, this test was performed to demonstrate the usefulness of the simulator and in addition to evaluate if the self-assembly aided by the gastric contraction deserves further investigation.

The diameter of the mock-ups is $11 \mathrm{~mm}$ and the length is $26 \mathrm{~mm}$, that is the size of commercial capsule endoscopes (Pill Cam SB ${ }^{\circledR}$, Given Imaging, Yoqneam, Israe) whose dimensions the ARES system is aiming at. Two magnets attached to the mock-ups are neodymium magnets (Q04x02x04Ni-N45, HKCM Engineering, Germany) and they are placed as MASH configuration as proposed in [15].

In the experiments, the silicone stomach model was filled with 0.7 litres of water and 5 mock-ups were dropped in it one by one. After self-assembly state without contraction was checked by a commercial endoscope as shown in Fig. 9, contractile motion using the mechanical setup was added for 5 minutes. The number of the connected mock-ups was counted before and after the contractile motion. This experiment was repeated 10 times.

\section{Results}

\subsection{Validation tests results}

The measured pressure variations of each section, the sum of pressure variations (calculated), and the measured pressure at the synchronized contraction are shown in Fig. 10.

The measured pressure at the synchronized contraction was up to $7 \mathrm{mmHg}$, which was in the range of human gastric pressure change, ranging from 3 up to $30 \mathrm{mmHg}$ as reported by Berne et al. [1]. Considering that the maximum pressure varies in each person, the developed model can serve as a reliable stomach simulator. The difference between the designed and measured values was mainly because the silicone thickness was too big to be contracted enough: on the contrary, the thickness cannot be smaller because a stomach with thinner wall can be easily torn. In addi-

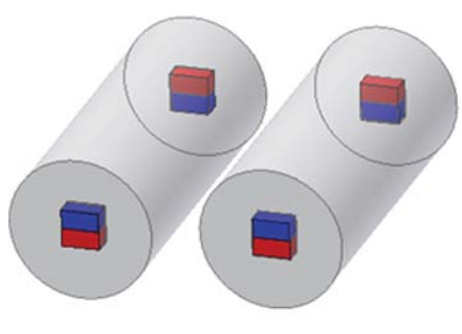

(a)

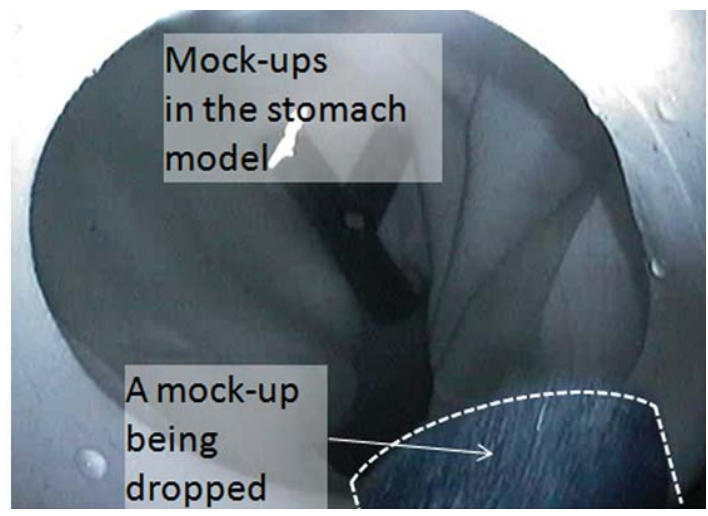

(b)

Fig. 9. (a) Design of the mock-up with two magnets. (b) Endoscopic view during the experiment. 


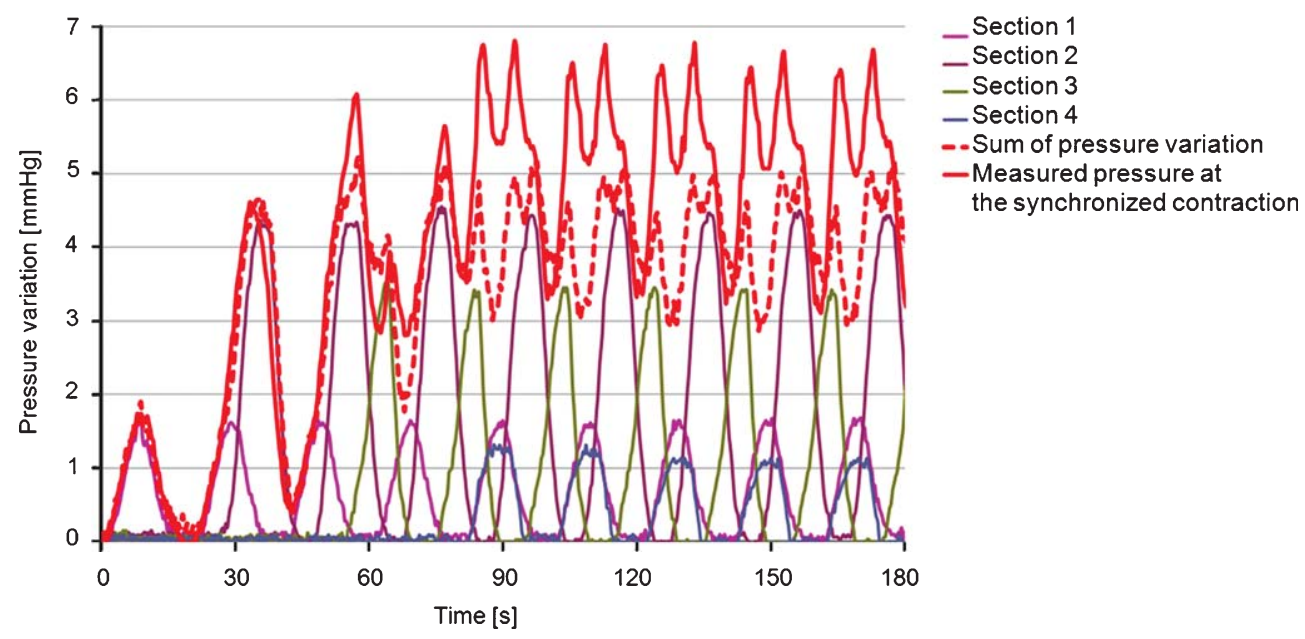

Fig. 10. The measured pressure variations of each section, the sum of pressure variations (calculated), and the measured pressure at the synchronized contraction.

tion, the wall needs to be thick enough to restore its deflated shape. Since the silicone is one of the best materials to replicate the intricate shape as the stomach, some compromises had to be undertaken for fabrication.

The periodic peaks of the pressure variation observed during the synchronized contraction can be explained by the sum of pressure variations, though the pressure variation at the synchronized contraction cannot simply be calculated as the linear sum of all contribution due to the complex shape of the stomach. The difference of the pressure variation, i.e. difference of the two line shapes (solid and dashed red lines of Fig. 10), is also due to the above mentioned problem.

In summary, the measured performance was not as precise as designed, but the fabricated simulator is capable of replicating the gastric contraction for the purpose of prototype testing and validation.

\subsection{Assembly tests results}

Table 2 shows the results of the self-assembly experiments. In the assembled topologies, five configurations statuses can be monitored: no assembly, assembly of two modules, assembly of three modules, assembly of four modules, and assembly of five modules.

Table 2 summarizes the configurations observed before and after 5 minutes of simulated contractile motion. The configurations of the mock-ups in the stomach model before and after the contractile motion are shown in Fig. 11 taking an example of the experiment No. 3. In the experiments No. 2, No. 3, No. 5, No. 7 and No. 9, the number of the assembled modules was increased after the contractile motion, and this suggests that the peristaltic activity can aid the magnetic selfassembly of the robotic capsules inside the stomach.

Table 2

Modules configurations before and after 5 minutes of contractile motion

\begin{tabular}{|c|c|c|c|c|c|c|c|c|c|c|}
\hline \multirow[b]{2}{*}{$\begin{array}{l}\text { Experiment } \\
\text { Number }\end{array}$} & \multicolumn{5}{|c|}{ Configuration of modules before the contractile motion } & \multicolumn{5}{|c|}{ Configuration of modules after contractile motion } \\
\hline & $\begin{array}{c}\text { No } \\
\text { assembly }\end{array}$ & $\begin{array}{c}\text { Two- } \\
\text { Assembled }\end{array}$ & $\begin{array}{c}\text { Three- } \\
\text { Assembled }\end{array}$ & $\begin{array}{c}\text { Four- } \\
\text { Assembled }\end{array}$ & $\begin{array}{c}\text { Five- } \\
\text { Assembled }\end{array}$ & $\begin{array}{c}\text { No } \\
\text { assembly }\end{array}$ & $\begin{array}{c}\text { Two- } \\
\text { Assembled }\end{array}$ & $\begin{array}{c}\text { Three- } \\
\text { Assembled }\end{array}$ & $\begin{array}{c}\text { Four- } \\
\text { Assembled }\end{array}$ & $\begin{array}{c}\text { Five- } \\
\text { Assembled }\end{array}$ \\
\hline No. 1 & 5 & & & & & 5 & & & & \\
\hline No. 2 & 3 & 1 & & & & 2 & & 1 & & \\
\hline No. 3 & 2 & & 1 & & & & 1 & 1 & & \\
\hline No. 4 & 1 & 2 & & & & 1 & 2 & & & \\
\hline No. 5 & 5 & & & & & 3 & 1 & & & \\
\hline No. 6 & 3 & 1 & & & & 3 & 1 & & & \\
\hline No. 7 & 5 & & & & & 3 & 1 & & & \\
\hline No. 8 & 5 & & & & & 5 & & & & \\
\hline No. 9 & 3 & 1 & & & & 2 & & 1 & & \\
\hline No. 10 & 5 & & & & & 5 & & & & \\
\hline
\end{tabular}




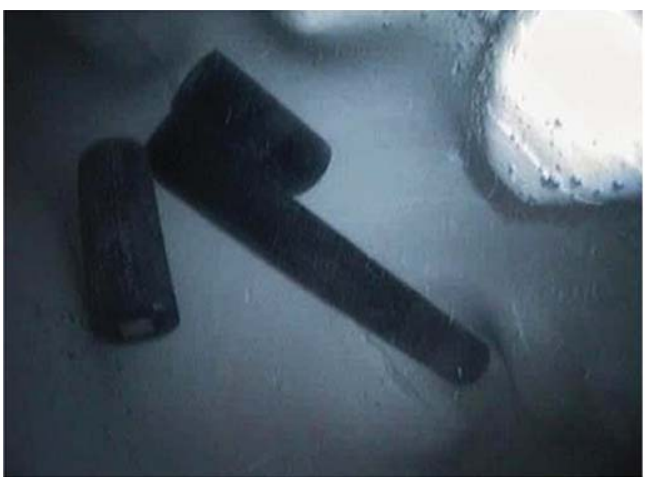

(a)

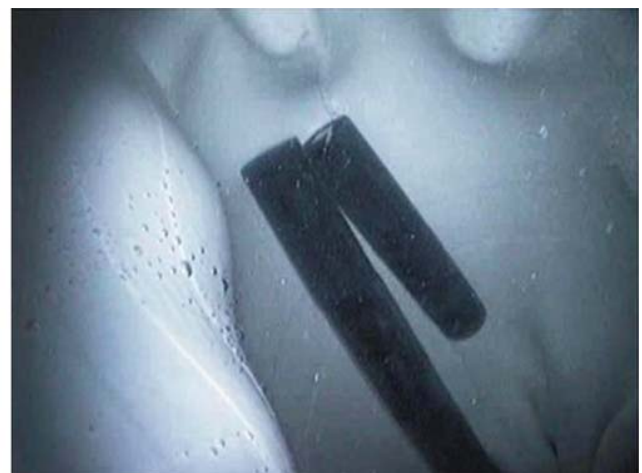

(b)

Fig. 11. Experiment No.3 showing assembled mock-ups in the stomach simulator: (a) before contraction (No-assembly: 2, three-assembled: 1) and (b) final configuration (Two-assembled: 1, Three-assembled: 1).

The experiments demonstrated that peristaltic motion may aid the magnetic self-assembly, and this topic deserves further investigation. This kind of experiments and evaluations can be realized only by the developed simulator.

\section{Conclusions}

A stomach simulator has been proposed for the analysis and validation of robotic devices for endoluminal tasks.

The silicone stomach model and the mechanical setup to simulate gastric contractile motion were designed and fabricated. The developed stomach simulator was validated and its usefulness was demonstrated by means of internal pressure measurements and selfassembly tests of mock-ups of robotic capsules.

The simulator would be useful for validating the performance during the prototyping of endoluminal robotic devices, and it would contribute to fast development and more quantitative assessment.

\section{Acknowledgements}

The authors would like to thank Professor Alfred Cuschieri for his medical consultancy. The authors are grateful to Mr. N. Funaro for the manufacturing of the prototypes. The work has been financed by the European Community projects "Ares" and "Araknes".

\section{References}

[1] R. Berne and M. Levy, Principi di fisiologia: Casa Editrice Ambrosiana (1996).

[2] A. Csendes and A.M. Burgos, Size, volume and weight of the stomach in patients with morbid obesity compared to controls, Obesity surgery 15(8) (2005), 1133-1136.

[3] I. De Zwart, B. Mearadji, H. Lamb, P. Eilers, A. Masclee A. De Roos, et al., Gastric motility: Comparison of assessment with real-time MR imaging or barostat measurement initial experience, Radiology 224(2) (2002), 592-597.

[4] J. Furness, The Enteric Nervous System, Wiley-Blackwell 2005.

[5] H. Gregersen, J. Barlow and D. Thompson, Development of a computer-controlled tensiometer for real-time measurements of tension in tubular organs, Neurogastroenterol Motil, 11(2) (1999), 109-118

[6] K. Harada, E. Susilo, A. Menciassi and P. Dario, Wireless reconfigurable modules for robotic endoluminal surgery, The IEEE International Conference on Robotics and Automation (ICRA) (2009), 2699-2704.

[7] X. He, J.H. Shin, H.-C Kim, C.W. Woo, S.H. Woo and W.C. Choi, et al., Balloon sheaths for gastrointestinal guidance and access: A preliminary phantom study, Korean journal of radiology : official,journal of the Korean Radiological Society 6(3) (2005), 167-172.

[8] J.A. Henry, G. O’Sullivan and A.S. Pandit, Using computed tomography scans to develop an ex-vivo gastric model, World Journal of Gastroenterology, 13(9) (2007), 1372-1377.

[9] G. Johnson, Holt Biology: Visualizing Life, Holt, Rinehart \& Winston, Orlando, 1994.

[10] B. Kim, S. Lee, J.H. Park and J.O. Park, Design and fabrication of a locomotive mechanism for capsule-type endoscopes using shape memory alloys (SMAs), IEEE-Asme Transactions on Mechatronics 10(1) (2005), 77-86.

[11] K. Koch, Clinical applications of electrogastrography, Chin Natl J Gastroenterol 2(1) (1996), 15-17.

[12] A. Lehman, M. Rentschler, S. Farritor and D. Oleynikov, The current state of miniature in vivo laparoscopic robotics, J Robotic Surg 1 (2007), 45-49.

[13] G.C. Matrone, C. Cipriani, E.L. Secco, G. Magenes and M.C. Carrozza, Principal components analysis based control of a 
multi-dof underactuated prosthetic hand, Journal of Neuroengineering and Rehabilitation 7 (2010).

[14] L. Mendoza, L. Leija, L. Garay and E.G. Ramos, Physical model of the stomach motor activity, 19th International Conference IEEE/EMBS, Chicago (1997).

[15] Z. Nagy, R. Oung, J.J. Abbott and N. BJ, Experimental Investigation of Magnetic Self-Assembly for Swallowable Modular Robots, Paper presented at the IEEE/RJS International Conference on Intelligent Robots and Systems (IROS), Nice, Franc (2008).

[16] A. Pal, K. Indireshkumar, W. Schwizer, B. Abrahamsson, M. Fried and J.G. Brasseur, Gastric flow and mixing studied using computer simulation, Proceedings of the Royal Society of London Series B-Biological Sciences 271(1557) (2004), 2587-2594.
[17] M. Quirini, A. Menciassi, S. Scapellato, C. Stefanini and P. Dario, Design and fabrication of a motor legged capsule for the active exploration of the gastrointestinal tract, IEEE-Asme Transactions on Mechatronics 13(2) (2008), 169-179.

[18] M. Sendoh, K. Ishiyama and K.I. Arai, Fabrication of magnetic actuator for use in a capsule endoscope, IEEE Transactions on Magnetics 39(5) (2003), 3232-3234.

[19] G. Tortora, P. Valdastri, E. Susilo, A. Menciassi, P. Dario and F. Rieber, et al., Propeller-based wireless device for active capsular endoscopy in the gastric district, Minimally Invasive Therapy \& Allied Technologies (MITAT) official journal of the Society for Minimally Invasive Therapy 18(5) (2009), 280-290. 

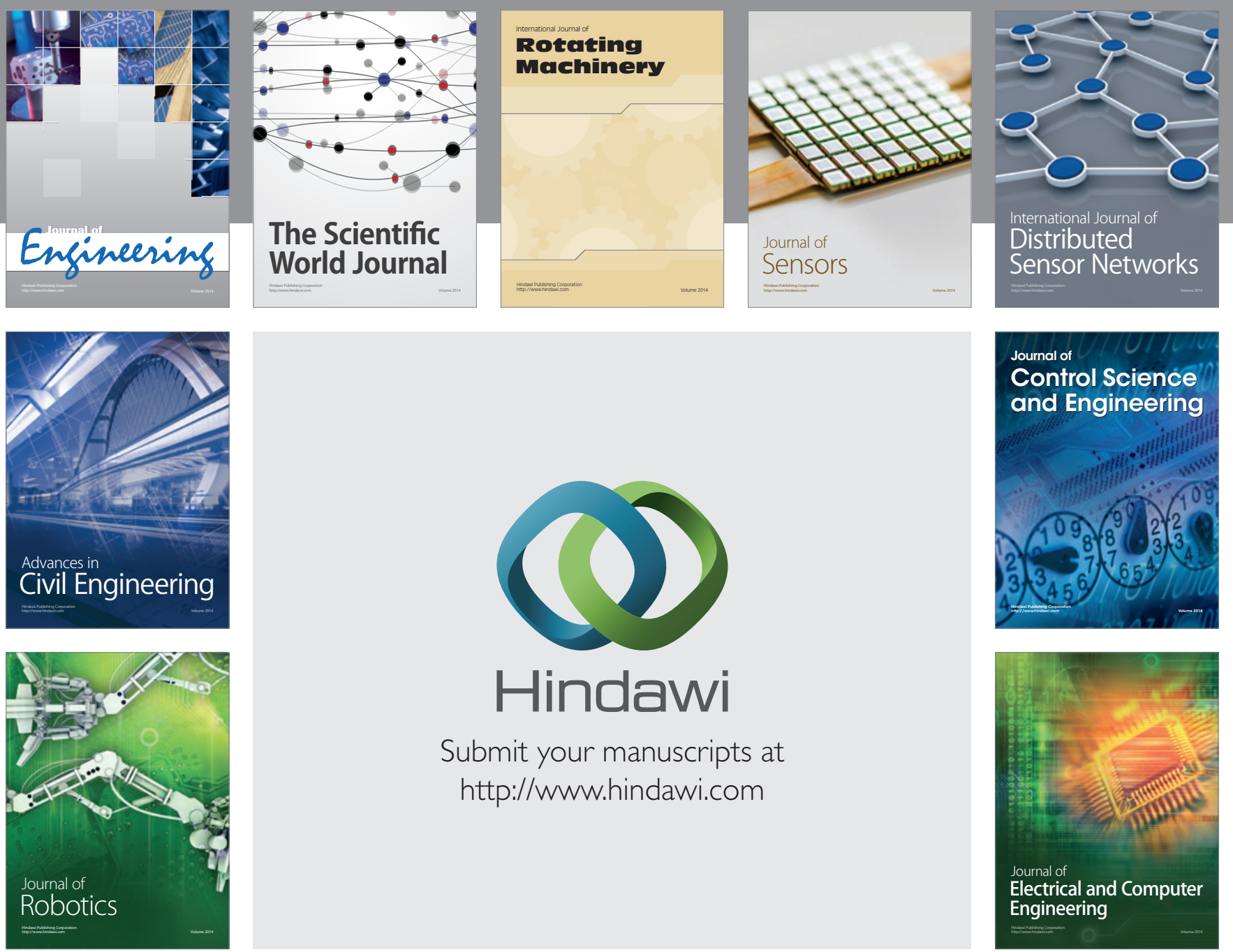

Submit your manuscripts at

http://www.hindawi.com
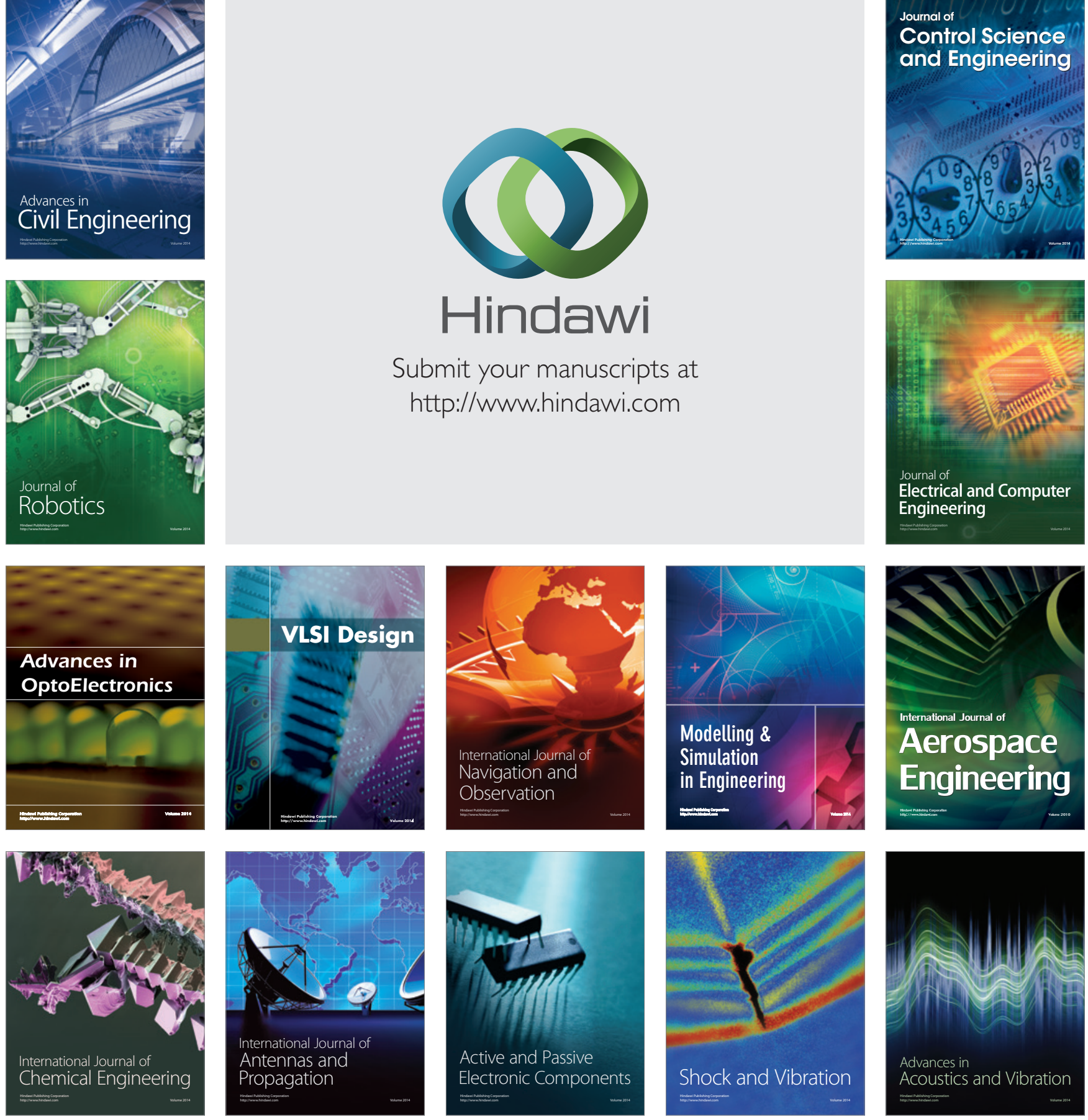